\section{Krebsschmerz: Hier sticht Akupunktur}

Krebspatienten mit Chemotherapie-induzierter Neuropathie (CIPN) profitieren von einer Schmerztherapie mit Akupunktur. In einer Studie mit elf CIPN-Patienten wurden sechs von ihnen innerhalb von drei Monaten mit 20 Nadeln behandelt. Die anderen erhielten keine Akupunktur. Ergebnis: In der Akupunkturgruppe erhöhte sich die Nervenleitgeschwindigkeit bei fünf der sechs Patienten. Diese berichteten auch von gelinderten Schmerzen. In der Kontrollgruppe reduzierten sich die Schmerzen nur bei zwei Patienten; die Leitgeschwindigkeit erhöhte sich nur bei einem. Ärzte Zeitung, 29.12.2011

\section{Querschnittslähmung: Stammzellen im Test}

Vier Patienten haben an der weltweit ersten genehmigten Studie zur Behandlung mit Stammzellen gegen Querschnittslähmung teilgenommen. Alle vier hatten zum Zeitpunkt der Behandlung unterhalb der Verletzung am Rückenmark keine motorischen und sensorischen Funktionen mehr. Das Präparat aus humanen embryonalen Stammzellen wurde den Patienten an der verletzten Stelle des Rückenmarks injiziert. Im Tierversuch erwies sich diese Behandlung bereits als erfolgreich: Zuvor gelähmte Mäuse konnten sich wieder bewegen. Ärzte Zeitung, 23.12.2011

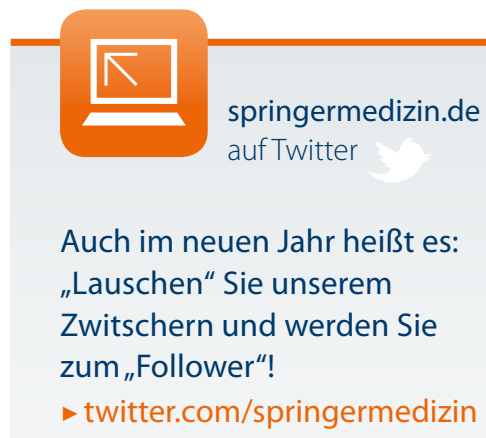

Harmlose Epileptiker, aber ...

\title{
Schneller kriminell durch Schädelhirntrauma
}

Patienten, die ein Schädelhirntrauma erleiden, werden danach signifikant häufiger kriminell als Gesunde - so die Ergebnisse einer Studie. Im Gegensatz dazu ist Epilepsie jedoch kein Risikofaktor für erhöhte Gewaltbereitschaft.

Mit 8,8\% liegt die Kriminalitätsrate nach Schädelhirntrauma (SHT) deutlich über dem Bevölkerungsdurchschnitt. Dies belegt die Auswertung schwedischer Klinikstatistiken der Jahre 1973 bis 2009. Die Daten von 22.914 hirnverletzten Patienten wurden mit denen von 22.947 Patienten verglichen, die an Epilepsie erkrankt waren. Fazit: Epilepsie scheint nicht zu einer erhöh- ten Rate an kriminellen Delikten, wie z.B. Mord, Überfall, Raub, Brandstiftung oder Sexualverbrechen zu führen, Hirnverletzungen wie z.B. Schädelhirntrauma dagegen sehr wohl. Vor allem die Schädigung des Frontalhirns erniedrigt die Hemmschwelle, da in dieser Region Funktionen wie Vernunft dominieren.

\section{Auch Vietnam-Veteranen betroffen}

Eine andere Studie kam zu einem ähnlichen Ergebnis: Ein Forscher, der 177 Vietnam-Veteranen mit Hirnverletzung untersuchte, berichtete ebenfalls über eine erhöhte Gewaltbereitschaft der Patienten.

$(c d)$

\section{Doch nicht so gutartig}

\section{Oberflächliche Thrombose kann Malignom bedeuten}

Tiefe Venenthrombosen und Lungenembolien können die Warnsignale einer okkulten Krebserkrankung sein. Oberflächliche Venenthrombosen galten in dieser Hinsicht bislang eher als unverdächtig. Zu Unrecht, wie eine dänische Registerstudie jetzt enthüllt.

Der Studie zufolge ist bei Patienten mit einer oberflächlichen Thrombophlebitis von einem ähnlich erhöhten Krebsrisiko auszugehen wie bei Patienten mit einer tiefen Venenthrombose (TVT) oder Lungenembolie (LE).

Ausgewertet werden konnten die Daten von 45.252 Patienten mit TVT, 24.332 mit LE und 7663 mit oberfläch- lichen Thrombosen. Die Rate an Krebsdiagnose im ersten Jahr nach dem Ereignis betrug 2,2\% nach einer oberflächlichen Venenthrombose, 2,7\% nach einer TVT und 2,9\% nach einer LE.

\section{Jede Thrombose hat es in sich}

Unabhängig von der Art der venösen Thromboembolie bestand die stärkste Assoziation mit Karzinomen der Leber, der Lunge, des Pankreas und der Ovarien sowie mit Non-Hodgkin-Lymphomen. Die Daten belegen, dass jegliche Venenthrombose „ein präklinischer Marker für eine Krebserkrankung “ ist, lautet das Fazit der Studienautoren. (bs) Eur J Cancer 2011 (online first) 